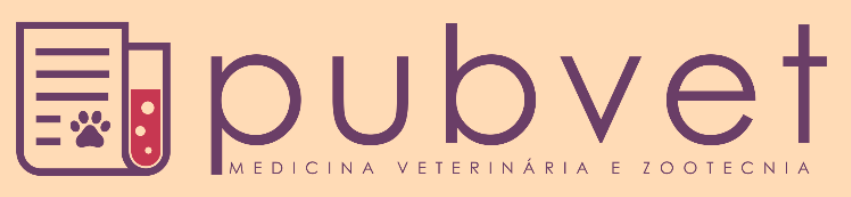

https://doi.org/10.31533/pubvet.v13n8a389.1-9

\title{
Hemangiossarcoma canino: revisão
}

\author{
Juliane Freitas $^{1 *}$, Lin Chieh Yi ${ }^{1}$, Gustavo Soares Forlani $^{2} \bullet$ \\ ${ }^{I}$ Discente do Curso de Medicina Veterinária da Universidade Comunitária de Chapecó - Unochapecó. Chapecó - SC Brasil. \\ ${ }^{2}$ Professor Doutor da Universidade Comunitária da Região de Chapecó-Unochapecó, Departamento de Medicina Veterinária-SC Brasil \\ *Autor para correspondência, E-mail: julianefreitas_vet@outlook.com
}

\begin{abstract}
Resumo. O hemangiossarcoma canino é uma neoplasia maligna de células endoteliais dos vasos sanguíneos, altamente metastáticos quando em sua forma visceral, podendo também se manifestar como forma primária cutânea. A espécie canina é descrita como sendo a mais afetada. Machos com idade entre dez e 12 anos são mais predispostos ao desenvolvimento do hemangiossarcoma (HSA). A manifestação clínica da doença é inespecífica e varia conforme a localização do sítio primário ou metástases. O diagnóstico definitivo ocorre através de histopatologia por biópsia do tumor. A terapia se dá por excisão cirúrgica e protocolos terapêuticos oncológicos. O prognóstico é considerado reservado, dependendo de qual fase foi diagnosticada a doença. Esta revisão de literatura tem por objetivo esclarecer e contribuir para o conhecimento da patologia, através da avaliação de órgãos e sistemas acometidos, sinais clínicos bem como apresentação de diagnósticos e tratamentos disponíveis.
\end{abstract}

Palavras chave: angiogênese, células endoteliais, hemangioma, oncologia veterinária

\section{Canine hemangiosarcoma: review}

Abstract. Canine Hemangiosarcoma is a malignant neoplasm of blood vessel endothelial cells, highly metastatic when in its visceral form, and may also manifest as a primary cutaneous form. The canine species is described as being the most affected. Males aged between 10 and 12 years are more predisposed to the development of Hemangiosarcoma (HSA). The clinical manifestation of the disease is nonspecific and varies according to the location of the primary site or metastases. The definitive diagnosis occurs through histopathology by biopsy of the tumor. The therapy is given by surgical excision and oncologic therapeutic protocols. The prognosis is considered reserved, depending on which phase the disease was diagnosed. This literature review aims to clarify and contribute to the knowledge of the pathology, through the evaluation of affected organs and systems, clinical signs as well as the presentation of available diagnoses and treatments.

Keywords: angiogenesis, endothelial cells, hemangioma, veterinary oncology

\section{Hemangiosarcoma canino: revisión}

Resumen. El Hemangiosarcoma (HSA) canino es una neoplasia maligna de células endoteliales de los vasos sanguíneos, altamente metastásica cuando está en su forma visceral, que también puede manifestarse como una forma cutánea primaria. La especie canina se describe como la más afectada.Los machos entre 10 y 12 años están más propensos al desarrollo del HSA. La manifestación clínica de la enfermedad es inespecífica y varía según la ubicación del sitio primario o metástasis. El diagnóstico definitivo se produce a través de la histopatología mediante la biopsia del tumor. La terapia se administra mediante diferentes procedimientos terapéuticos, quirúrgicos y oncológicos. El pronóstico 
se considera reservado, dependiendo de la etapa en la que se diagnosticó la enfermedad. Esta revisión de la literatura tiene como objetivo aclarar y contribuir al conocimiento de la patología, a través de la evaluación de los órganos y sistemas afectados, signos clínicos, así como la presentación de diagnósticos y tratamientos disponibles

Palabras clave: angiogénesis, células endoteliales, hemangioma, oncología veterinaria

\section{Introdução}

O hemangiossarcoma (HSA) se apresenta como uma neoplasia extremamente invasiva e metastática, acometendo qualquer órgão vascularizado por ser derivado de alterações de crescimento de células com linhagem endotelial. Sua característica agressiva se deve à rápida disseminação das células tumorais pela via hematógena (Flores et al., 2012; Guberman et al., 2015), cujo sítio primário comum é o baço. Todavia, podem ocorrer no fígado, pulmões, coração e rins. Cães são mais acometidos que as demais espécies, apresentando sinais clínicos inespecíficos e variados de acordo com a localização. Existem raças susceptíveis ao desenvolvimento da patologia como Pastor Alemão, Beagle, Bulldog, Pointer Inglês, Golden Retriever, Labrador, Boxer e Dálmatas (Chipunza et al., 2005; Ferraz et al., 2008). Apesar de incomum, o HSA pode se manifestar na forma cutânea, como sítio primário ou metastático, podendo acometer a derme ou subcutâneo, com predileção da pele abdominal, prepucial e os membros pélvicos. Sugere-se que cães de pelo curto ou de pele pouco pigmentado e submetidos à exposição excessiva a raios ultravioletas estão mais predispostos, desencadeando a variante primária da doença (Camboim et al., 2017; Filgueira et al., 2012; Soares et al., 2017).

O diagnóstico definitivo é obtido por exames histopatológicos de amostras do tumor primário ou metástases. A terapêutica inicial baseia-se no reestabelecimento do paciente, garantindo que o mesmo esteja apto à realização de procedimentos mais invasivos, como a excisão cirúrgica do tumor, quimioterapias e demais protocolos oncológicos (Frenz et al., 2014; Jericó et al., 2015).

Considerando a alta ocorrência de hemangiossarcomas em cães de diversas raças, idades e diferentes órgãos acometidos, a realização desta revisão bibliográfica tem por objetivo esclarecer aspectos clínicos, de diagnóstico e de tratamento do hemangiossarcoma canino, a fim de contribuir para um melhor entendimento da patologia aos interessados.

\section{Epidemiologia}

O HSA possui maior prevalência na espécie canina quando comparado às demais espécies domésticas, sobretudo animais com idade entre oito e catorze anos, sendo mais encontrado em machos e raças predispostas como Pastor Alemão, Labrador e Golden Retriever. O baço, o átrio direito, o tecido subcutâneo, o fígado e o espaço retro perineal são locais comumente envolvidos, sendo que tumores no baço representam metade da porcentagem total, seguido do átrio direito, do tecido subcutâneo, do fígado e simultaneamente em outros locais. Nos caninos, representa $2 \%$ dos tumores, sendo 45 a $51 \%$ tumores malignos esplênicos (Clifford et al., 2000; Withrow et al., 2014).

\section{Etiologia}

O hemangiossarcoma (HSA) é classificado como uma neoplasia maligna e agressiva de caráter altamente metastática, com sua origem a partir do endotélio vascular, e seu alvo, portanto, se dá em qualquer órgão vascularizado. O comportamento biológico do tumor de HSA possui diversas formas que são infiltrativas e dão origem a metástases precocemente na doença; com exceção às formas cutâneas, conjuntivais e de terceira pálpebra, pois estes possuem baixos potenciais metastáticos (Frenz et al., 2014; Lamerato-Kozicki et al., 2006). Uma das hipóteses para a ontogênese do HSA é a ocorrência de mutações das células endoteliais vasculares diferenciadas que as tornam com potencial maligno, outra, por uma parada na diferenciação das células estaminais hemangioblásticas. Já a expressão que pode estar relacionada com a proliferação tumoral se dá pela elevação de fatores angiogênicos obtidos pela análise imunohistoquímica; fatores pelos quais a proliferação e a regressão de células endoteliais são rigidamente controladas por mecanismos fisiopatológicos sob diversas condições de angiogênese. O primeiro fator é o crescimento endotelial vascular (VEGF), que são variações de células produzidas em resposta a inúmeras situações e, dentre elas, fatores de crescimento e mutação de oncogênese 
(Lamerato-Kozicki et al., 2006). O segundo é o fator básico de crescimento fibroblástico (FGF) que, junto com a VEGF, compõe a família dos receptores da tirosina quinase, que possuem papel importante na regulação da angiogênese, ou seja, afetam diretamente no crescimento e desenvolvimento tumoral (Frenz et al., 2014; Lamerato-Kozicki et al., 2006; Murakami et al., 2008). Os fatores de crescimento citado anteriormente desempenham papel fundamental de proliferação, crescimento, desenvolvimento e diferenciação celular embrionária de um organismo durante toda sua vida; importantes também como promotores de crescimento tumoral, pois interrompem o equilíbrio entre a proliferação e morte celular em células neoplásicas (Halper, 2010).

\section{Fisiopatogenia}

Segundo Fosmire et al. (2004), histologicamente o HSA é um tumor que pode apresentar morfologia heterogênea dentro da mesma massa neoplásica. Além disso, o tumor se apresenta de forma não encapsulado, mal demarcado e apresenta áreas difusas de hemorragia e necrose. O tecido é composto por células endoteliais imaturas ovóides a fusiformes que se arranjam em camadas sólidas formando canais rudimentares tortuosos contendo hemácias, e podem ser compostos de uma ou mais camadas de células endoteliais pleomórficas com núcleos hipercromáticos e citoplasma abundante. As células são invasivas para o parênquima adjacente. Os HSA apresentam uma lesão única com diferentes tamanhos e cores, variando de cinza- claro a vermelho-escuro e são nodulares e macios; a ruptura da cápsula que reveste o parênquima tumoral associada à hemorragia é frequentemente observada (Withrow et al., 2014).

As síndromes paraneoplásicas podem estar presentes em um paciente com HSA. Estas síndromes são manifestações clínicas que produzem alterações de modo indireto pelas células tumorais que, em condições normais, não o fariam. Como consequência, há produção de substâncias biologicamente ativas que não estão associadas à invasão direta de neoplasias ou metástases, sendo estas substâncias os hormônios e seus precursores, os fatores de crescimento e as interleucinas. Além disso, as síndromes podem afetar o sistema imunológico, causando imunossupressão. Sua presença reduz a qualidade de vida e o tempo de sobrevivência dos pacientes acometidos, portanto, deve-se interromper esse processo o quanto antes. Dentre as alterações, podemos citar as hematológicas, as endócrinas, as neurológicas e as cutâneas (Clifford et al., 2000; Fosmire et al., 2004; Murakami et al., 2008).

As alterações hematológicas frequentemente relatadas em animais com HSA são a anemia, a trombocitopenia e a coagulação intravascular disseminada (CID). A anemia é definida como uma redução das hemácias, hematócrito e hemoglobina no sangue periférico, resultado de sangramento intracavitário e/ou hemólise micro angiopática (HMA). A trombocitopenia, comum em neoplasias que atingem o baço, é descrito como uma redução de plaquetas no sangue que, nesse caso, é secundário à HMA. Por fim, a coagulação intravascular disseminada (CID), também chamada de coagulopatia de consumo se manifesta no animal por sangramento espontâneo (Jericó et al., 2015).

A CID é uma síndrome secundaria a inúmeras patologias, inclusive as neoplasias. Em pacientes oncológicos ocorre o aumento da trombina e fibrina em consequência da estimulação tumoral. A coagulação disseminada nesses pacientes é estimulada pela produção de proteínas pró-coagulante pelas células tumorais, além do fato, do tumor iniciar a agregação plaquetária promovendo a formação de coágulos e posteriormente um quadro de diátese hemorrágica levando o paciente a óbito. Com exceção da trombocitopenia, os outros dois são comuns em cães com HSA independentemente da localização primária do tumor ou do estágio (Jericó et al., 2015; Vital, 2014).

\section{Sinais clínicos}

Conhecido pela sua agressividade, os HSA produzem metástases precocemente e são evidentes na clínica e pode ocorrer por via hematógena ou transcelômica por ruptura esplênica. Os órgãos mais frequentemente metastatizados são o fígado, o omento, o mesentério e o pulmão. No entanto, não é patognomônico de um animal com lesões hepáticas por HSA apresentar metástases hepáticas (Clifford (Clifford et al., 2000; Withrow et al., 2014). Os quadros clínicos apesentados estão normalmente relacionados com o local de origem do tumor primário e se há presença de metástases ou ruptura do tumor. Neste último caso, os animais se apresentam com quadro grave de hemorragia interna, percebido pela distensão abdominal e manifestação de sinais associados à perda aguda de sangue. Animais que estão anêmicos observam-se as mucosas hipocoradas, taquicardia e taquipneia. Quando se suspeita de 
presença de metástase no miocárdio, há presença de arritmias, intolerância a exercícios, dispneia, ascite e diminuição de sons cardíacos e pulso jugular. No geral, o paciente apresenta letargia, sinais de depressão, anorexia, massa abdominal palpável, hemoperitônio, anemia e choque (Clifford et al., 2000; Jericó et al., 2015; Withrow et al., 2014).

\section{Diagnóstico}

O diagnóstico precoce do hemangiossarcoma é de suma importância para o sucesso do tratamento. A detecção da doença já nos primeiros sinais possibilita a melhor efetividade das terapias, aumentando as taxas de sobrevivências e diminuindo as taxas de disseminação do tumor. Os animais avaliados clinicamente podem apresentar outros sintomas que não estão envolvidos diretamente com o tumor, mas sim com a morbidade causada por ele. Muitas vezes o paciente é acometido de diferentes patologias em um mesmo momento, quando as neoplasias estão envolvidas nesse curso as complicações são maiores, devido ao alto comprometimento fisiológico induzido pelo tumor (Fernandes \& Nardi, 2017; Yamamoto et al., 2013).

No decorrer da vida clínica dos animais, os seus tutores devem ser orientados sobre as possibilidades do desenvolvimento de neoplasias. Estes devem estar atentos a quaisquer alterações seja comportamental ou física em seu animal, devendo realizar check up semestrais ou anuais. Os exames mais indicados para triagem oncológica incluem os exames laboratoriais de hemograma, dosagens bioquímicas de ureia, creatinina, TGP, fosfatase alcalina, albumina; avaliação cardiológica com a mensuração da pressão arterial, eco e eletrocardiograma e avaliação endócrina com dosagens bioquímicas de glicose, triglicérides e colesterol. Assim, rastreando dados indicativos de neoplasias sistêmicas ou locais. É de responsabilidade do médico veterinário durante consultas a avaliação de locais onde as neoplasias ocorrem com maiores frequências (Ministérioa da Saúde, 2004; Kealy et al., 2012).

Pelo conhecimento da prevalência de determinado tumor juntamente aos diagnósticos diferenciais que excluem outras patologias, é possível estabelecer a etiologia da neoplasia e proceder com a terapia o mais rápido possível. Basicamente, o diagnóstico de hemangiossarcomas ocorre através do histórico clínico, anamnese, raça, idade, exame físico, hemograma completo, análise bioquímica sérica, exame de urina, radiografias torácicas, ultrassonografias abdominais e o diagnóstico definitivo é dado de exames histopatológicos de amostras obtidas por biópsia (Ministério da Saúde, 2004).

Durante a avaliação clínica pode ser observado sinais inespecíficos como apatia, abdômen alongado, frequências cardíaca e respiratória aumentados, mucosas claras e emagrecimento. A ocorrência desses episódios é observada por longos períodos, até que ocorra o reestabelecimento do animal. O abdômen distendido pode ser evidenciado por quadros geralmente consequentes da ascite. Na auscultação podem ocorrer arritmias e abafamento dos sons ou efusão pericárdica. A presença de relevante intolerância a exercícios pode levar a morte súbita quando não houver o repouso recomendado. Em casos de HSA cutâneo, pode haver a presença de nódulos geralmente discretos, firmes e com coloração semelhante a hematomas traumáticos, muitas vezes confundidos com tais (Ministério da Saúde, 2004; Kealy et al., 2012; Yamamoto et al., 2013). Em hemangiossarcomas viscerais podem ocorrer colapsos de órgãos acometidos, causando hemorragias intracavitárias que levam a quadros de anemias regenerativas. Há a possibilidade de ocorrer perdas sanguíneas abruptas, plaquetopenia e coagulação intravascular disseminada (Vital, 2014).

Segundo Ferraz et al. (2008), a trombocitopenia (plaquetopenia) pode ser observada em 30 a $60 \%$ dos cães com HSA. As causas de hemácias nucleadas no sangue incluem infiltração de células malignas na medula óssea, hematopoiese extramedular, hipoxemia e hipoesplenismo associado com infiltração neoplásica. As alterações bioquímicas estarão relacionadas com as possíveis metástases ou locais do tumor primário, podendo incluir elevação das enzimas hepáticas, quadros de hipoalbuminemia e hipoglobulinemia, aumento de alanina aminotransferase, fosfatasse alcalina, uréia e creatinina (Filgueira et al., 2012; Kealy et al., 2012). Exames de imagem auxiliam na identificação de estruturas neoformadas na cavidade abdominal e torácica, indicando tumores primários e/ou metástases. A ultrassonografia abdominal é muito útil no estadiamento das neoplasias; a imagem em tempo real possibilita avaliar a ecogenicidade da lesão, o envolvimento dos linfonodos bem como a verificação do comprometimento de órgãos adjacentes, possibilitando um prognóstico mais preciso para remoção cirúrgica. Em casos de suspeita de envolvimento cardíaco deve-se realizar o ecodopplercardiograma, que possibilita a pesquisa 
acerca da presença de trombos, alterações anatômicas nas diferentes cavidades do coração, análise do fluxo sanguíneo, a capacidade funcional e a direção seguida juntamente com as válvulas cardíacas. A associação do Doppler na avaliação de massas tumorais permite a identificação de áreas necróticas, permitindo maior segurança na realização de biópsia ecodirigida (Vital, 2014).

Para obter um diagnóstico confiável da patologia é necessária a realização de exames de biópsia. Esses podem ser realizados com auxílio da imagem ecográfica do ultrassom, evitando procedimentos diagnósticos mais invasivos, como a laparotomia ou a toracotomia exploratória. A realização da punção por agulha fina guiada por ultrassom (PAAF) permite a obtenção de material para a citologia celular, sendo esse um procedimento válido para o diagnóstico de HSA, já que não requer incisão cirúrgica e, em alguns casos, é possível obter o resultado diagnóstico no mesmo dia. Todavia, em casos de efusões torna-se difícil a diferenciação celular e resultados inconclusos pelo fato da agulha não remover quantidades de tecido suficientes. A biópsia realizada com agulha cortante (Trucut) fornece fragmentos maiores de tecido, permitindo o aumento da especificidade diagnóstica; porém, têm-se riscos maiores de complicações já que necessita ser feita sob anestesia e em ambiente ambulatorial (Kealy et al., 2012).

Histologicamente o hemangiossarcoma é caracterizado por células neoplásicas pleomórficas, formando espaços vasculares indistintos associados à hemorragia e necrose. É através do exame histopatológico realizado com material de biópsia ou excisão das lesões que se estabelece o diagnóstico definitivo de hemangiossarcoma. Juntamente com os demais dados de exames listados, é possível estagiar o tumor através da escala TNM e estabelecer o tratamento (Filgueira et al., 2012).

O estadiamento clínico tumoral é realizado através do Sistema TNM (T: tumor primário; N: linfonodo; M: metástase) da Organização Mundial de Saúde (OMS) desenvolvido por Pierre Denoix (França). O sistema é baseado na localização anatômica, tamanho do tumor, extensão clínica e patológica, envolvimento de linfonodos, sinais de metástase e é aplicável à HSA canina (Filgueira et al., 2012; Jericó et al., 2015; Kealy et al., 2012). O modelo de estadiamento tumoral é descrito na tabela 1.

Tabela 1. Estadiamento clínico dos HSA em cães de acordo com a Organização Mundial da Saúde

\begin{tabular}{lll}
\hline Estadiamento & Característica do Estágio & Classificação do Estágio \\
\hline & Tumor Primário $(T)$ & \\
& T0: sem evidência do tumor & \\
Estágio I & T1: tumor menor que 5 cm de diâmetro e confinado ao local primário ou derme & T0 ou T1, N0, M0 \\
& T2: tumor maior ou igual a 5 cm ou ulcerado; invasão de tecidos subcutâneos & II: T1 ou T2, N0 ou N1, M0 \\
& T3: tumor invadindo estruturas adjacentes; invasão da musculatura & \\
Estágio II & Nonfonodos regionais $(N)$ & N1: envolvimento de linfonodos regionais \\
& N2: envolvimento de linfonodos a distância & T2 ou T3, N0, N1 ou N2, M1 \\
\hline & Metástases distantes $(M)$ & \\
\hline
\end{tabular}

Fonte: Adaptado de Fernandes \& Nardi (2017).

\section{Tratamento}

Os hemangiossarcomas são classificados como Tumores Malignos de Partes Moles, o estadiamento da doença juntamente com a realização ou não de quimioterapia são os pontos cruciais e determinantes de sobrevida do animal, sendo que quando mais próximo ao estágio III, menores são as expectativas de sobrevida. $\mathrm{O}$ tratamento de cães com hemangiossarcoma baseia-se principalmente na ressecção cirúrgica total do tumor primário, realizada de forma primária, com objetivo de possibilitar um maior índice de cura. No entanto, em razão do rápido desenvolvimento das metástases, em geral, o procedimento cirúrgico não interfere no tempo de sobrevida dos pacientes, devendo ser associado à quimioterapia e imunoterapia adjuvante (Yamamoto et al., 2013).

Os pacientes submetidos à cirurgia devem ter acompanhamento clínico com a solicitação de exames pré-operatórios como o hemograma completo, bioquímica hepática e renal, coagulograma e monitorados 
por eletrocardiograma antes, durante e após a cirurgia. Esses pacientes são pré-dispostos a desenvolver coagulação intravascular disseminada e arritmias ventriculares no pós-operatório. A técnica cirúrgica deve complementar uma boa margem de segurança, com o objetivo da completa excisão do tumor e de tecidos adjacentes (Fernandes \& Nardi, 2017).

A quimioterapia no tratamento de hemangiossarcomas é capaz de prolongar a sobrevida dos cães. Os agentes antineoplásicos exercem atividades de interferir na síntese de DNA ou em sua função; na síntese de RNA e proteínas. O objetivo da terapia antineoplásica em medicina veterinária é paliativa e visa produzir a remissão e/ou conforto para o paciente, melhorar a sua qualidade de vida e reduzindo assim sintomas causados por metástases ou tumores inoperáveis (Medeiros Júnior \& Cordovani, 2006). A combinação de agentes quimioterápicos vem sendo estudada amplamente e demonstra maior efetividade que a utilização de agentes únicos e isolados (Jericó et al., 2015). A quimioterapia metronômica é uma opção terapêutica que potencializa a resposta imunitária antitumoral e diminui a densidade vascular tumoral. O protocolo consiste na administração contínua de doses fixas e baixas de quimioterápicos sem interrupções prolongadas no tratamento, diferentemente da terapia quimioterápica convencional aonde são administradas altas doses com longos intervalos (Elmslie et al., 2008). O objetivo da dosagem metronômica é baseado no fato de que as células endoteliais são muito mais sensíveis à exposição contínua a baixas doses de drogas quimioterápicas e menos propensas a sofrer mutações genéticas do que as células tumorais, que tipicamente desenvolvem rápida resistência a drogas (Shimizu \& Oku, 2004). Os protocolos terapêuticos denominados de VAC citam o uso de fármacos do grupo dos antibióticos antraciclinas (doxorrubicina) como agente único ou associado aos inibidores de microtúbulos (vincristina) e agentes alquilantes (ciclofosfamida).

Estudos relatam que cães tratados com protocolo VAC após a cirurgia possuem uma sobrevida média de 172 dias, e para pacientes tratados apenas com a cirurgia de 19 a 65 dias (Ferraz et al., 2008). O esquema de administração para os protocolos VAC I e II é demonstrado na tabela 2.

Tabela 2. Esquema de administração do protocolo VAC I e II em cães.

\begin{tabular}{|c|c|c|c|}
\hline \multirow[b]{2}{*}{ Dia } & \multicolumn{3}{|c|}{ Protocolo VAC I } \\
\hline & Doxorrubicina & Vincristina & Ciclofosfamida \\
\hline $1^{\circ}$ & $25 \mathrm{mg} / \mathrm{m}^{2} \mathrm{EV}$ & $0,7 \mathrm{mg} / \mathrm{m}^{2} \mathrm{EV}$ & - \\
\hline $8^{\circ}$ ao $11^{\circ}$ & - & - & $50 \mathrm{mg} / \mathrm{m}^{2} \mathrm{VO} / 24 \mathrm{hrs}$ \\
\hline $15^{\circ}$ ao $17^{\circ}$ & - & - & $50 \mathrm{mg} / \mathrm{m}^{2} \mathrm{VO} / 24 \mathrm{hrs}$ \\
\hline $22^{\circ}$ & Repetir todo ciclo, num total de seis vezes & & \\
\hline
\end{tabular}

\begin{tabular}{|c|c|c|c|}
\hline \multirow[b]{2}{*}{ Dia } & \multicolumn{3}{|c|}{ Protocolo VAC II } \\
\hline & Doxorrubicina & Ciclofosfamida & Vincristina \\
\hline $1^{\mathrm{o}}$ & $\begin{array}{l}30 \mathrm{mg} / \mathrm{m}^{2} \mathrm{EV} \text { (até dez quilos) } 25 \mathrm{mg} / \mathrm{m}^{2} \mathrm{EV} \text { (menor } \\
\text { ou igual a dez quilos) } 1 \mathrm{mg} / \mathrm{m}^{2} \mathrm{EV} \text { (menos de dez } \\
\text { quilos) }\end{array}$ & $200 \mathrm{mg} / \mathrm{m}^{2} \mathrm{VO}$ ou EV & - \\
\hline $8^{\circ}$ & - & - & $0,7 \mathrm{mg} / \mathrm{m}^{2} \mathrm{EV}$ \\
\hline $15^{\circ}$ & - & - & $0,7 \mathrm{mg} / \mathrm{m}^{2} \mathrm{EV}$ \\
\hline $22^{\circ}$ & Repetir todo ciclo acima, num total de 4 a 6 vezes & & \\
\hline
\end{tabular}

Fonte: Adaptado de Ferraz et al. (2008).

Os profissionais atuantes no tratamento quimioterápico de animais devem estar cientes dos riscos à saúde causada pela manipulação desses agentes citotóxicos. As medidas de segurança devem ser criteriosamente alinhadas, utilizando métodos preventivos e de apoio como a cabine de fluxo laminar, avental impermeável de mangas longas, luvas descartáveis, óculos protetores e máscaras faciais respiratórias que contenham filtros HEPA (Hayes, 2007; Jericó et al., 2015; Medeiros Júnior \& Cordovani, 2006).

A utilização de inibidores das cicloxigenases 2 (COX-2) é proposta como parte do tratamento antineoplásico, apresentando um papel limitador na carcinogênese - mecanismo de ação descrito pela diminuição da apoptose celular, aumento da atividade metaloproteinases, aumento do metabolismo dos xenobióticos (Hayes, 2007). Entre as novas opções terapêuticas incluem-se a utilização de 
imunomoduladores, inibidores da angiogênese e eletroquimiotarapia. A imunoterapia como opção terapêutica na oncologia tem sido explorada e estudada em cães com hemangiossarcomas. $\mathrm{O}$ uso de imunoestimulantes como o muramil tripéptido fosfatidiletanolamina liposomal encapsulado (L-MTPPE) em terapias adjuvantes de quadros de neoplasias, possibilita um aumento da habilidade de macrófagos e monócitos em reconhecer e destruir células neoplásicas e um aumento da concentração plasmática do fator de necrose tumoral e de outras citocinas, promovendo assim atividade antimetástatica em tumores malignos. A associação de imunoestimuladores juntamente com protocolos antineoplásicos apresenta uma melhoria significativa de sobrevivência, devido ao fato do L MTP-PE encapsular-se em lipossomos, aumentando a endocitose e a captação tecidual de MTP-PE por células de defesa e prolongando sua meia vida na circulação (Barros \& Repetti, 2015; Kahn et al., 2013). Os inibidores da metaloproteinase de matriz e da angiogênese são usados para bloquear os fatores que estimulam a formação de novos vasos e moléculas que permitem a formação de vasos neoformados, causando a incapacitação de novas células endoteliais em divisão (Gibot et al., 2013). Alguns dos inibidores conhecidos da angiogênese incluem angiostatina, endostatina, esteróides angiostáticos, interferons, interleucina-12, ácido retinóico e inibidores teciduais de metaloproteína-matriz (Dennis et al., 2011). A eletroquimiotarapia é uma técnica física que permite que as moléculas citotóxicas sejam eficientemente liberadas em células tumorais por induzir permeabilização da membrana plasmática ao quimioterápico local ou sistêmico, melhorando o tratamento local de tumores cutâneos e subcutâneos com grande eficácia. O tratamento é utilizado como técnica paliativa (Bertazzolo et al., 2005).

\section{Prognóstico}

Fatores prognósticos são características patológicas e biológicas do paciente e de sua patologia que permitem prever a evolução e seu tempo de sobrevida. Inúmeros fatores prognósticos são importantes para avaliar a resolução ou evolução de muitas neoplasias. Os tópicos incluem o tipo histológico e grau histológico, morfologia, índice mitótico, tamanho do tumor, localização, invasão e padrão das margens cirúrgicas, localização primária e idade do paciente. De um modo geral, pelo fato do HSA ser uma neoplasia maligna e agressiva, o prognostico é considerado reservado, sendo que em animais com presença de metástases a excisão cirúrgica do tumor primário é apenas um tratamento paliativo (Bertazzolo et al., 2005; Martins et al., 2013).

\section{Considerações finais}

O HSA é uma neoplasia maligna de prognóstico reservado. A realização de exames complementares é fundamental para estabelecer a melhor conduta terapêutica para cada paciente, sendo normalmente preconizada a realização da exérese da massa tumoral com ampla margem de segurança associada ou não a protocolos com fármacos antineoplásicos. $\mathrm{O}$ emprego dessas diretrizes é fundamental para promover melhora da qualidade e da expectativa de vida dos animais com essa afecção.

\section{Referências bibliográficas}

Barros, V. T. M. \& Repetti, C. S. F. (2015). Metronomic chemotherapy in dogs: a review. Revista Portuguesa de Ciência Veterinárias, 110593-594.

Bertazzolo, W., Dell'Orco, M., Bonfanti, U., Ghisleni, G., Caniatti, M., Masserdotti, C., . . . Roccabianca, P. (2005). Canine angiosarcoma: cytologic, histologic, and immunohistochemical correlations. Veterinary Clinical Pathology, 34(1):28-34.

Camboim, A. S., Benvenutti, M. E. M., Oliveira, E. L., Vaz, A. F. M., Silva, R. M. N., Dantas, A. F. M. \& Souza, A. P. (2017). Manifestação de síndrome paraneoplásica em um cão com hemangiossarcoma cutâneo: relato de caso. Brazilian Journal of Veterinary Medicine, 39(2):126-132.

Chipunza, J., Chimonyo, M., Bhebhe, E., Mukaratirwa, S. \& Chitanga, S. (2005). Canine cutaneous neoplasms: prevalence and influence of age, sex and site on the presence and potential malignancy of cutaneous neoplasms in dogs from Zimbabwe. Journal of the South African Veterinary Association, 76(2):59-62.

Clifford, C. A., Mackin, A. J. \& Henry, C. J. (2000). Treatment of canine hemangiosarcoma: 2000 and beyond. Journal of Veterinary Internal Medicine, 14(5):479-485. 
Dennis, M. M., McSporran, K. D., Bacon, N. J., Schulman, F. Y., Foster, R. A. \& Powers, B., E. (2011). Fatores prognósticos para sarcomas cutâneos e subcutâneos de tecido mole em cães. Patologia Veterinária, 48(1):73-84.

Elmslie, R. E., Glawe, P. \& Dow, S. W. (2008). Metronomic therapy with cyclophosphamide and piroxicam effectively delays tumor recurrence in dogs with incompletely resected soft tissue sarcomas. Journal of Veterinary Internal Medicine, 22(6):1373-1379.

Fernandes, S. C. \& Nardi, A. D. B. (2017). Hemangiossarcomas. In C. R. Nardi \& A. D. B. Nardi (Eds.), Oncologia em cães e gatos (pp. 431-445). Rio de Janeiro, Brasil: Editora Roca.

Ferraz, J. R. S., Roza, M. R., Caetano Júnior, J. \& Costa, A. C. (2008). Hemangiossarcoma canino: revisão de literatura. Jornal Brasileiro de Ciência Animal, 1(1):35-48.

Filgueira, K. D., Reis, P. F. C. C., Batista, J. S. \& Paula, V. V. (2012). Hemangiossarcoma cutâneo com metástase no sistema nervoso central de um canino. Acta Scientiae Veterinariae, 40(1):1-7.

Flores, M. M., Panziera, W., Kommers, G. D., Irigoyen, L. F., Barros, C. S. L. \& Fighera, R. A. (2012). Aspectos epidemiológicos e anatomopatológicos do hemagiossarcoma em cães: 40 casos (19652012). Pesquisa Veterinária Brasileira, 32(12):1319-1328.

Fosmire, S. P., Dickerson, E. B., Scott, A. M., Bianco, S. R., Pettengill, M. J., Meylemans, H., . . Getzy, D. M. (2004). Canine malignant hemangiosarcoma as a model of primitive angiogenic endothelium. Laboratory Investigation, 84(5):562-572.

Frenz, M., Kaup, F.-J. \& Neumann, S. (2014). Serum vascular endothelial growth factor in dogs with haemangiosarcoma and haematoma. Research in Veterinary Science, 97(2):257-262.

Gibot, L., Wasungu, L., Teissié, J. \& Rols, M.-P. (2013). Antitumor drug delivery in multicellular spheroids by electropermeabilization. Journal of Controlled Release, 167(2):138-147.

Guberman, Ú. C., Merlini, N. B., Perches, C. S., Fonzar, J. F., Sereno, M. G., Mamprim, M. J., . . . Brandão, C. V. S. (2015). Hemangiossarcoma corneal em cão. Arquivo Brasileiro de Medicina Veterinária e Zootecnia, 67(2):343-346.

Halper, J. (2010). Growth factors as active participants in carcinogenesis: a perspective. Veterinary Pathology, 47(1):77-97.

Hayes, A. G. (2007). Cancer, cyclo-oxygenase and nonsteroidal anti-inflammatory drugs-can we combine all three? Veterinary and Comparative Oncology, 5(1):1-13.

Jericó, M. M., Kogika, M. M. \& Andrade Neto, J. P. (2015). Tratado de medicina interna de cães e gatos. Rio de Janeiro, Brasil: Guanabara Koogan.

Kahn, S. A., Mullin, C. M., Lorimier, L.-P., Burgess, K. E., Risbon, R. E., Fred III, R. M., . . Clifford, C. A. (2013). Doxorubicin and deracoxib adjuvant therapy for canine splenic hemangiosarcoma: a pilot study. The Canadian Veterinary Journal, 54(3):237.

Kealy, J. K., McAllister, H. \& Graham, J. P. (2012). Radiologia e Ultrassonografia do Cão e do Gato (Vol. 1). São Paulo: Manole.

Lamerato-Kozicki, A. R., Helm, K. M., Jubala, C. M., Cutter, G. C. \& Modiano, J. F. (2006). Canine hemangiosarcoma originates from hematopoietic precursors with potential for endothelial differentiation. Experimental Hematology, 34(7):870-878.

Martins, B. D. C., Torres, B. B. J., Rodriguez, A. A. M., Gamba, C. O., Cassali, G. D., Lavalle, G. E., . . Melo, E. G. (2013). Clinical and pathological aspects of multicentric hemangiosarcoma in a Pinscher dog. Arquivo Brasileiro de Medicina Veterinária e Zootecnia, 65(2):322-328.

Medeiros Júnior, L. C. \& Cordovani, P. (2006). Diagnóstico por imagem na pesquisa de metástases de neoplasias mamárias. Revista Nosso Clínico, 9(50):20-28.

Ministério da Saúde [Secretaria de Atenção à Saúde]. Instituto Nacional de Câncer. TNM: classificação de tumores malignos / traduzido por Ana Lúcia Amaral Eisenberg. 6. ed. - Rio de Janeiro: INCA, 2004. 254p. Saúde [acesso em abril 2019]. Disponível em: http://bvsms.saude.gov.br/bvs/publicacoes/inca/tnm2.pdf 
Murakami, M., Sakai, H., Kodama, A., Mori, T., Maruo, K., Yanai, T. \& Masegi, T. (2008). Expression of the anti-apoptotic factors Bcl-2 and survivin in canine vascular tumours. Journal of Comparative Pathology, 139(1):1-7.

Shimizu, K. \& Oku, N. (2004). Terapia anti-angiogênica para câncer. Biological and Pharmaceutical Bulletin, 27599-605.

Soares, N. P., Medeiros, A. A., Szabó, M. P. J., Guimarães, E. C., Fernandes, L. G. \& Santos, T. R. (2017). Hemangiomas e hemangiossarcomas em cães: estudo retrospectivo de 192 casos (20022014). Ciência Animal Brasileira, 18(e-30889):1-10.

Vital, A. F. (2014). Distúrbios hemostáticos em cães com neoplasia: revisão de literatura. Medicina Veterinária, 8(1):12-19.

Withrow, S. J., Page, R. \& Vail, D. M. (2014). Small animal clinical oncology. St. Louis, USA: Elsevier Health Sciences.

Yamamoto, S., Hoshi, K., Hirakawa, A., Chimura, S., Kobayashi, M. \& Machida, N. (2013). Epidemiological, clinical and pathological features of primary cardiac hemangiosarcoma in dogs: a review of 51 cases. Journal of Veterinary Medical Science, 75(11):1433-1441.

Recebido: 15 de julho, 2019.

Aprovado: 7 de agosto, 2019.

Publicado: 10 de setembro, 2019.

Licenciamento: Este artigo é publicado na modalidade Acesso Aberto sob a licença Creative Commons Atribuição 4.0 (CC-BY 4.0), a qual permite uso irrestrito, distribuição, reprodução em qualquer meio, desde que o autor e a fonte sejam devidamente creditados. 